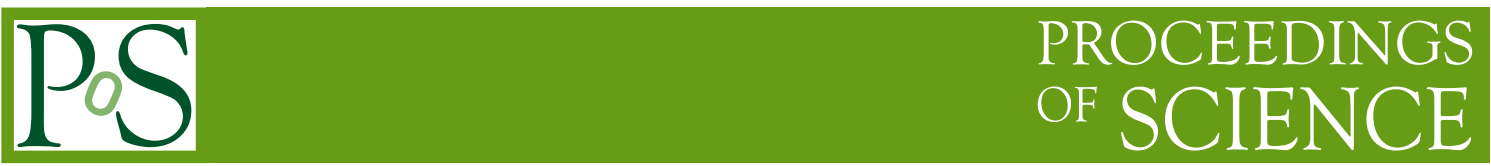

\title{
Neutrino decay processes and flavour oscillations
}

\author{
Alexey Lickunov, ${ }^{a}$ Konstantin Stankevich, ${ }^{a, b, *}$ Alexander Studenikin ${ }^{a, c}$ and Maxim \\ Vyalkov $^{a}$ \\ ${ }^{a}$ Faculty of Physics, Lomonosov Moscow State University, Moscow 119991, Russia \\ ${ }^{b}$ Kotel'nikov Institute of Radio Engineering and Electronics RAS, 125009, Moscow, Russia \\ ${ }^{c}$ Joint Institute for Nuclear Research, Dubna 141980, Moscow Region, Russia \\ E-mail: lichkunov.aa15@physics.msu.ru, kl.stankevich@physics.msu.ru, \\ studenik@srd.sinp.msu.ru, vvialkov.mm17@physics.msu.ru
}

We further develop the theory of the neutrino flavour oscillations accounting for the quantum decoherence effect engendered by the neutrino radiative decay in an external electromagnetic field. In particular, we have considered the most general form of the neutrino electromagnetic vertex and obtained in the explicit form the dissipative term in the neutrino master equation. It shown that the dissipative term depends on the neutrino radiative decay rates.

*** The European Physical Society Conference on High Energy Physics (EPS-HEP2021), ***

*** 26-30 July $2021 * * *$

*** Online conference, jointly organized by Universität Hamburg and the research center DESY ***

${ }^{*}$ Speaker 
In our previous studies [1-4] in the derivation of the neutrino oscillation probabilities we have developed a new formalism, based on quantum electrodynamics of open systems [5], to account the effect of quantum decoherence due to the neutrino radiative decay $v \rightarrow v+\gamma$ in a specific case of the electron media [6]. In this paper we generalize our consideration and show that the developed formalism can be applied to account for the neutrino radiative decay in an external electromagnetic field in the case of the most general neutrino-photon couplings. In particular, we consider the neutrino electromagnetic vertex in the form [7]

$$
\Lambda_{\mu}^{k j}(q)=\left(\gamma_{\mu}-q_{\mu} \gamma_{\nu} q_{\nu} / q^{2}\right)\left[f_{Q}^{k j}\left(q^{2}\right)+f_{A}^{k j}\left(q^{2}\right) q^{2} \gamma_{5}\right]-i \sigma_{\mu \nu} q^{\nu}\left[f_{M}^{k j}\left(q^{2}\right)+i f_{E}^{k j}\left(q^{2}\right) \gamma_{5}\right],
$$

where $q$ is the four-momentum of the photon, $f_{Q}^{k j}, f_{A}^{k j}, f_{M}^{k j}, f_{E}^{k j}$ are the neutrino charge, anapole, dipole magnetic and electric form factors. It is considered that neutrino millichages, charge radii, dipole magnetic and electric moments can contribute to the radiative decay rate.

We start with the quantum Liouville equation for the density matrix $\rho$ of a system composed of neutrinos and an external electromagnetic field

$$
\frac{d \rho}{d t}=-i\left[H_{v}+H_{\text {int }}(t), \rho\right],
$$

where $H_{v}$ is the nonradiative neutrino evolution Hamiltonian. The Hamiltonian $H_{\text {int }}$ describes the interaction of neutrinos with the radiative and external electromagnetic fields,

$$
H_{\text {int }}(x)=\sum_{\mu} j_{\alpha}(x) A^{\mu}(x),
$$

where $A^{\mu}$ is the electromagnetic field and $j_{\mu}$ is the neutrino current

$$
j_{\mu}(x)=\sum_{k, j} \bar{v}_{k}(x) \Lambda_{\mu}^{k j} v_{j}(x)
$$

Here $v_{k}(x)$ is the neutrino mass state and $\Lambda_{\mu}^{k j}$ is the neutrino electromagnetic vertex given by Eq.(1).

The Liouville Eq.(2) describes the evolution of the complex system composed of neutrinos and photons. In order to extract the neutrino evolution we follow the approach developed in [1]. At the first stage, we exclude the environment evolution tracing out (2) the electromagnetic degrees of freedom and get

$$
\rho_{v}\left(t_{f}\right)=\operatorname{tr}_{E} \rho\left(t_{f}\right)=\operatorname{tr}_{E}\left(\operatorname{Texp}\left[\int_{t_{i}}^{t_{f}} d^{4} x\left[H_{\text {int }}(x), \rho\left(t_{i}\right)\right]\right]\right),
$$

where $\rho_{v}$ is the density matrix for the neutrino subsystem. Following the prescription described in details in [1] we arrive to the master equation for the neutrino subsystem

$$
\frac{d \rho_{v}}{d t}=-i\left[H_{v}, \rho_{v}\right]+D\left[\rho_{v}\right]
$$

The first term on the right hand side of (6) describes the neutrino evolution given by the nonradiative neutrino Hamiltonian $H_{v}$. The second term is the dissipative operator in the Lindblad form $[8,9]$ 
that appears due to the neutrino interaction with photons

$$
\begin{array}{r}
D\left[\rho_{\nu}\right]=\sum_{\omega_{n}} \Gamma\left(\omega_{n}\right)\left(1+N\left(\omega_{n}\right)\right)\left(j\left(\omega_{n}, \vec{k}\right) \rho_{\nu} j^{\dagger}\left(\omega_{n}, \vec{k}\right)-\frac{1}{2}\left\{j^{\dagger}\left(\omega_{n}, \vec{k}\right) j\left(\omega_{n}, \vec{k}\right), \rho_{v}\right\}\right)+ \\
+\sum_{\omega_{n}} \Gamma\left(\omega_{n}\right) N\left(\omega_{n}\right)\left(j^{\dagger}\left(\omega_{n}, \vec{k}\right) \rho_{v} j\left(\omega_{n}, \vec{k}\right)-\frac{1}{2}\left\{j\left(\omega_{n}, \vec{k}\right) j^{\dagger}\left(\omega_{n}, \vec{k}\right), \rho_{v}\right\}\right),
\end{array}
$$

where $\omega_{n}$ is the energy difference between neutrino states participating in the radiative decay and $N\left(\omega_{n}\right)$ is the Planck distribution function

$$
N\left(\omega_{n}\right)=\frac{1}{e^{\beta \omega_{n}}-1} .
$$

Here $\beta$ is the temperature of the photons.

The neutrino decoherence parameters are defined by the neutrino decay rates $\Gamma\left(\omega_{n}\right)$. In equation (7) we have decomposed the neutrino current (3)

$$
j(t, \vec{k})=\sum_{\omega_{n}} e^{-i \omega_{n} t} j\left(\omega_{n}, \vec{k}\right),
$$

on the eigenoperators of the neutrino Hamiltonian

$$
\left[H_{\nu}, j\left(\omega_{n}, \vec{k}\right)\right]=\omega_{n} j\left(\omega_{n}, \vec{k}\right)
$$

where $\vec{k}$ is the neutrino momentum.

The first term in Eq. (7) is responsible for the spontaneous and thermally induced emission processes and the second one is responsible for the thermally induced absorption processes. Note that the dissipative term (7) is proportional to the neutrino radiative decay rates $\Gamma\left(\omega_{n}\right)$. Thus the obtained results enable one to follow the influence of the neutrino radiative decays through the neutrino quantum decoherence effect on flavour and spin-flavour oscillations.

This research has been supported by the Interdisciplinary Scientific and Educational School of Moscow University "Fundamental and Applied Space Research" and also by the Russian Foundation for Basic Research under Grant No. 20-52-53022-GFEN-a. The work of KS is also supported by the RFBR under grant No. 20-32-90107 and by the "BASIS" Foundation No. 20-2-2-3-1. The work of MV is also supported by the "BASIS" Foundation No. 20-2-1-25-1.

\section{References}

[1] K. Stankevich, A. Studenikin, Neutrino quantum decoherence engendered by neutrino radiative decay, Phys. Rev. D 101 (2020) 056004.

[2] K. Stankevich, A. Studenikin, Neutrino evolution and quantum decoherence, J. Phys. Conf. Ser. 1468 (2020) 012148.

[3] K. Stankevich, A. Studenikin, Neutrino decoherence due to radiative decay, PoS ICHEP2018 (2019) 925. 
[4] K. Stankevich, A. Studenikin, Neutrino quantum decoherence due to entanglement with a magnetic field, PoS EPS-HEP2017 (2018) 645.

[5] H.P. Breuer, F. Petruccione, "The theory of open quantum systems" (Oxford, UK: Univ. Pr. (2002) $625 \mathrm{p}$ ).

[6] J. C. D’Olivo, J. F. Nieves and P. B. Pal, Phys. Rev. Lett. 64 (1990), 1088 doi:10.1103/PhysRevLett.64.1088

[7] C. Giunti and A. Studenikin, Rev. Mod. Phys. 87 (2015), 531 doi:10.1103/RevModPhys.87.531 [arXiv:1403.6344 [hep-ph]].

[8] G. Lindblad, On the Generators of Quantum Dynamical Semigroups, Commun. Math. Phys. 48 (1976) 119.

[9] V. Gorini, A. Kossakowski, E. Sudarshan, Completely Positive Dynamical Semigroups of N Level Systems, J. Math. Phys. 17 (1976) 821. 